MATEC Web of Conferences 37, 01064 (2015)

DOI: $10.1051 /$ matecconf $/ 20153701064$

C) Owned by the authors, published by EDP Sciences, 2015

\title{
PARAMETRIC IDENTIFICATION OF CONTROL SYSTEMS USING ARTIFICIAL NEURAL NETWORKS
}

\author{
Andrey A. Zakamaldin ${ }^{1 a}$, Vladimir S. Andyk ${ }^{1}$ \\ ${ }^{1}$ National Research Tomsk Polytechnic University, 634050 Tomsk, Russia
}

\begin{abstract}
For the problem of parametric identification of control objects proposed a method based on the use of artificial neural network feedforward type a perceptron. This method allows us to estimate the parameters of the mathematical model of the system with a maximum error of $2.8 \%$ and significantly reduce the time of the identification procedure.
\end{abstract}

\section{Introduction}

Nowadays it pays great attention to the quality control of technical systems, because control objects are becoming harder and smarter. To improve the quality control mechanisms used costly, modern high-order control device, various regulators. But the high possibility of such devices can be put into practice only in the presence of automatic settings, allowing the system to adapt to changes in the parameters of the control object.

Current methods setting regulator usually includes the identification procedure of the control object. The problem of identification in this case is solved in real time, which makes high demands on the accuracy and speed of the algorithms. However, the classical methods of solving this problem require many experiments on control object.

In order to reduce the duration of identification to the lowest possible value equal to the time of the transition process in the system, it is proposed to use an artificial neural network (ANN), analyzing the dynamic characteristics of the control object [1].

\section{Statement of the problem and description of the method of identification}

According to the proposed method, the structure of the mathematical model is defined by a priori known information. Processes occurring in the control object, for example, can be represented as second order differential equation [2].

$$
a_{2} y^{\prime \prime}(t)+a_{1} y^{\prime}(t)+a_{0} y(t)=b_{0} x(t)
$$

The expression for the transient response of the system depends on the type of roots of the characteristic equation. Consideration will be given only one task, if the roots of this equation are real

${ }^{a}$ Corresponding author : dron.and1@yandex.ru

This is an Open Access article distributed under the terms of the Creative Commons Attribution License 4.0, which permits unrestricted use, distribution. and reproduction in anv medium, provided the original work is properlv cited. Article available at nttp://www.matec-conerences.org or nttp://dx.dol.org/10.1051/mateccont/20153/01064 
and distinct, since the proposed method of solution is applicable to other cases. Then the equation of the system can be written as

$$
T_{1} T_{2} y^{\prime \prime}(t)+\left(T_{1}+T_{2}\right) y^{\prime}(t)+y(t)=k x(t)
$$

The transient response

$$
y(t)=k \cdot\left(1-\frac{T_{1}}{T_{1}-T_{2}} \cdot e^{-\frac{t}{T_{1}}}+\frac{T_{2}}{T_{1}-T_{2}} \cdot e^{-\frac{t}{T_{2}}}\right)
$$

Schedule, investigated the transient response is shown in Fig. 1.

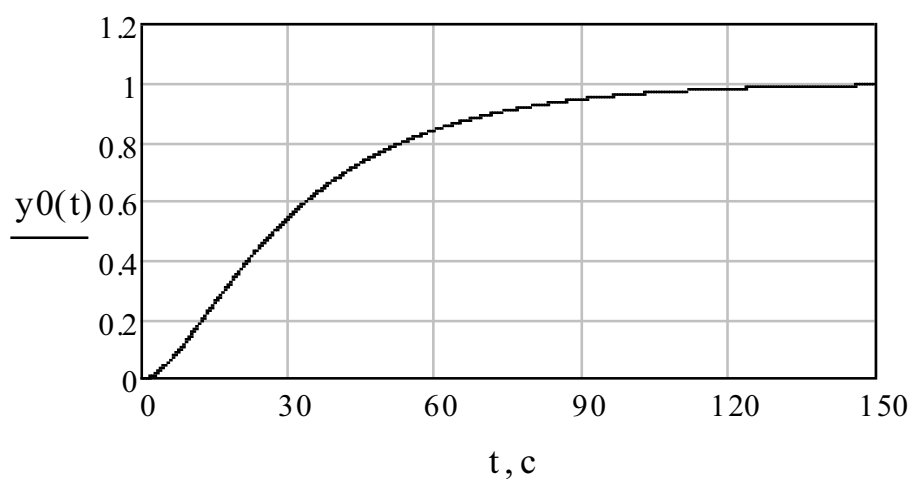

Figure 1. The transient response of the system under specified conditions $k=1, T_{01}=7, T_{02}=28$

The task is to identify the parameters $T_{1}$ and $T_{2}$, i.e. in their quantification.

As shown by the analysis $[3,4,5,6]$ to solve the problem of identification can be used as direct distribution ANN (sigmoidal, radial), and network with feedback. For further research it was chosen a simpler structure of direct distribution network (feedforward). An example of such a network is a multilayer perceptron (feed-forward backprop). ANN scheme, organized in the software package MATLAB R2012a Neural Network Toolbox, is presented in Fig. 2.

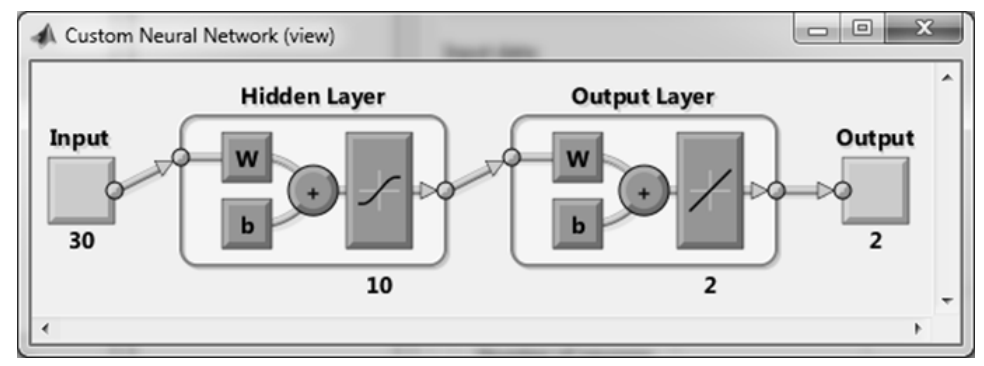

Figure 2. Structure of the ANN

The structure of the ANN for solving the problem of identification was formed as follows. The dimension of the input vector (Input) network corresponds to the number of samples of the transient response of the object: $\mathrm{N}=30$. The dimension of the output vector (Output Layer, Output) network is determined by the number of object parameters: $M=2$. The number of neurons in the hidden layer Nc may be is taken from the condition: 


$$
N_{c} \leq \frac{\left(N_{p}-1\right) \cdot M}{N+M+1}=\frac{(200-1) \cdot 2}{30+2+1} \approx 12
$$

$\mathrm{Np}$ - number of training examples (sample size).

The optimum size of a neural network learning corresponds to the maximum capacity, and therefore the best adequacy of the resulting model. Therefore, the most efficient number of neurons determined experimentally in this case was made $\mathrm{Nc}=10$ with activation functions 'tansig' (hyperbolic tangent), the output layer comprising two neurons with linear activation function 'purelin' (Fig. 2).

ANN training was conducted using the Levenberg - Marquardt (trainlm). It should be noted that the function 'trainlm' is used by default, because it provides the fastest training, but requires a lot of RAM. The method is efficient, providing the completion of training for 347 epochs of training with a mean-square error of not more than 0.069 . The criterion for stopping the training was higher limits 'validation check' $=200$. That is, the learning process is terminated automatically when the validation error for the sample exceeds the lowest for 200 epochs. Further training will be retraining the network, since the error on the test set begins to increase, and the error on the training set continues to decrease (Fig. 3).

\section{Best Validation Performance is $\mathbf{0 . 0 6 9 4 4 8}$ at epoch 347}

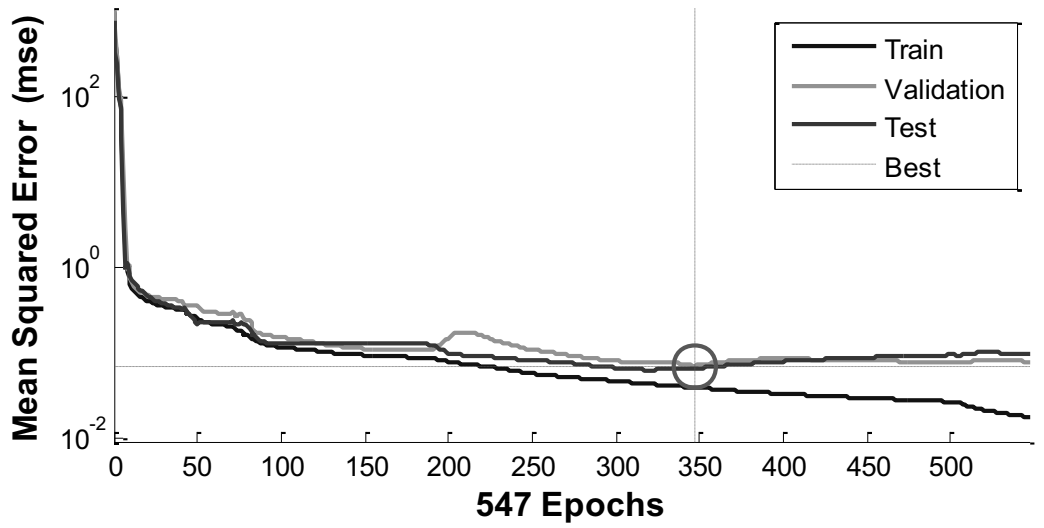

Figure 3. The process of learning network (dependent mean square error for the training, validation, test sets from the steps of training)

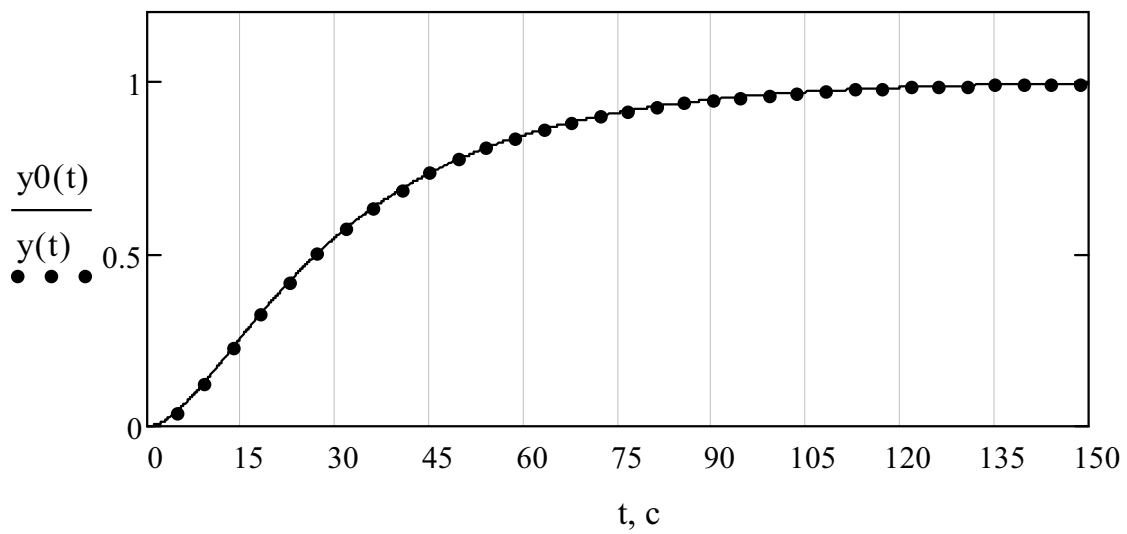

Figure 4. Comparison of the original data with the results of identification 


\section{The results of the identification}

Estimate the quality of identification by the degree of divergence of the coefficients obtained by the INS and the original; on the degree of divergence transient characteristics of the original system (y0) and the resulting model (y). Figure 4 shows the transients for the system with parameters: $T_{01}=7, T_{02}=28$ and obtained by simulation on the ANN system with parameters: $T_{1}=6.9053$, $T_{2}=28.0479$. Analysis of the curves confirms the adequacy of parametric identification of neural network method.

When testing the ANN trained on the dataset transient response revealed that the maximum relative error in determining the parameters of the system is $\sim 2.8 \%$.

\section{Conclusions}

Simulation is recommended to start with a simple classical models, and only then move on to the neural networks. If the non-linear model (neural network), realizing a highly nonlinear mapping of input signals into output, summarizes worse than a simpler model, it indicates that it is inadequate. And, without even realizing neural model may be the best solution to this problem, but the lack of sample size or excessive size, complexity ANN cannot adequately adjust the neural network. There is no correct neural modeling methodology to construct a neural network, in other words there is no strict sequence to the constructed model has optimum properties.

It should be noted that the model used for the consideration of the problem is very simple in structure, and by learning algorithms. Quantification parameters for the second-order system can be considered sufficiently accurate and adequate.

Known methods of parametric identification of the considered system, for example, the method of Oldenburg, Sartorius [2], the method of identification in the frequency domain on the amplitude spectrum of the output signal, various analytical methods, of course, have a place to be. For ideal transmission characteristics, mathematical models, obtained by these methods are quite adequate. But it should be noted that these methods are less effective in relation to the control of complex objects. Characteristics obtained with such object are not clearly defined character of technological process, and it can change parameters over time. Thus, each of the mathematical method of estimation have the field where his model is adequate, and much more narrow field where the method allows to get good grades.

\section{References}

1. Anisimov, M. Grachev, Vestnik ISPU, 3, 55 (2008)

2. V. Andyk, Automatic Control Theory (Tomsk Polytechnic University, Tomsk, 2005)

3. S. Shtovba, Design of fuzzy systems by means of MATLAB (Moscow, Goryachaya liniya Telekom, 2007)

4. A. Rothstein, Intellectual identification technology: fuzzy logic, genetic algorithms, neural networks (Vinnitsa, UNIVERSUM-Vinnitsa, 1999)

5. C. Bailer-Jones, D. MacKay, P. Withers, Network: Computation in Neural Systems, 9 (4), 531 (1998)

6. G. Kerschen, K. Worden, A. Vakakis, J. Golinval, Mechanical Systems and Signal Processing 20, 505 (2006) 